\title{
Characteristics and Treatment Trends for Emphysematous Pyelonephritis in Korea: A 10-Year Multicenter Retrospective Study
}

\author{
Seung-Kwon Choi, Koo Han Yoo' ${ }^{1}$, Jeong Woo Lee ${ }^{1,2}$, Seung II Jung ${ }^{3}$, Eu Chang Hwang ${ }^{3}$, Joongwon Choi ${ }^{4}$, Woong Bin Kim ${ }^{5}$, \\ Jung Sik Huh ${ }^{6}$, Jin Bong Choi ${ }^{7}$, Yeonjoo Kim ${ }^{8}$, Jae Min Chung, , Ju-Hyun Shin ${ }^{10}$, Jae Hung Jung ${ }^{11}$, Hong Chung ${ }^{12}$, \\ Sangrak Bae ${ }^{13}$, Tae-Hyoung Kim ${ }^{14}$ \\ Department of Urology, Seoul Medical Center, ${ }^{1}$ Department of Urology, College of Medicine, Kyung Hee University, Seoul, ${ }^{2}$ Department of \\ Urology, Dongguk University College of Medicine, Gyeongju, ${ }^{3}$ Department of Urology, Chonnam National University Medical School, \\ Gwangju, ${ }^{4}$ Department of Urology, VHS Medical Center, Seoul, ${ }^{5}$ Department of Urology, Soonchunhyang University College of Medicine, \\ Bucheon, ${ }^{6}$ Department of Urology, Jeju National University College of Medicine, Jeju, ${ }^{7}$ Department of Urology, College of Medicine, \\ The Catholic University of Korea, Seoul, ${ }^{8}$ Department of Urology, Daegu Fatima Hospital, Daegu, ${ }^{9}$ Department of Urology, Pusan National \\ University Yangsan Hospital, Yangsan, ${ }^{10}$ Department of Urology, Chungnam National University College of Medicine, Daejeon, ${ }^{11}$ Department \\ of Urology, Yonsei University Wonju College of Medicine, Wonju, ${ }^{12}$ Department of Urology, Konkuk University School of Medicine, Chungju, \\ ${ }^{13}$ Department of Urology, Uijeongbu St. Mary's Hospital, College of Medicine, The Catholic University of Korea, Uijeongbu, ${ }^{14}$ Department of \\ Urology, Chung-Ang University College of Medicine, Seoul, Korea
}

Purpose: This study examined the characteristics, current treatment trends, and outcomes of patients with emphysematous pyelonephritis (EPN) in Korea.

Materials and Methods: Two hundred and seventeen patients diagnosed with EPN were evaluated using abdominal computed tomography in 2011-2021 at 15 institutes in Korea. The patients' demographic and clinical characteristics, treatment modalities, and treatment outcomes were analyzed. The total study period was divided arbitrarily into groups A (2011-2014), B (2015-2017), and C (2018-2021) to analyze the trends in the EPN treatment.

Results: The mean age of the patients was 65.1 years; there were more female patients $(74.2 \%)$ than male patients. The overall mortality rate was $10.6 \%$. Ninety-five (43.8\%), 98 (45.2\%), and $24(11.0 \%)$ patients were treated with medical, minimally invasive, and surgical management, respectively; the corresponding mortality rates were $13.7 \%, 6.1 \%$, and $16.7 \%$. There was no significant change in the proportion of patients treated with medical management over time (group $\mathrm{A}=46.5 \%$, group $\mathrm{B}=47.0 \%$, and group $\mathrm{C}=38.8 \%$ ). The proportion of patients treated with minimally invasive management gradually increased over time (group $A=35.2 \%$; group $B=43.9 \%$; group $C=55.0 \%$ ), while those who underwent surgical management decreased gradually over time (group $A=18.3 \%$, group $B=9.1 \%$, and group $C=6.3 \%$ ). No differences in mortality rates were observed between the groups.

Conclusions: EPN with medical and minimally invasive management had a relatively high treatment success rate, which increased gradually, while surgical management decreased gradually over time in Korea. The mortality rate was relatively lower than that reported in studies published before the 2010 s.

Keywords: Pyelonephritis; Therapeutics; Treatment outcome; Mortality; Republic of Korea

Copyright $\odot$ 2021, Korean Association of Urogenital Tract Infection and Inflammation. All rights reserved. (c) (1) (s) This is an open access article distributed under the terms of the Creative Commons Attribution Non-Commercial License (http://creativecommons.org/licenses/by-nc/4.0) which permits unrestricted non-commercial use, distribution, and reproduction in any medium, provided the original work is properly cited.
Received: 11 August, 2021

Revised: 20 August, 2021

Accepted: 20 August, 2021
Correspondence to: Koo Han Yoo

(iD) https://orcid.org/0000-0001-7952-7902

Department of Urology, Kyung Hee University Hospital at Gangdong, College of Medicine, Kyung Hee University, 892 Dongnam-ro, Gangdong-gu, Seoul 05278, Korea

Tel: +82-2-440-6271, Fax: +82-2-440-7744

E-mail: yookoohan@khu.ac.kr 


\section{INTRODUCTION}

Emphysematous pyelonephritis (EPN) is an acute, severe, necrotizing renal infection characterized by gas production in the renal parenchyma, collecting system, or perinephric tissue [1]. Kelly and MacCallum [2] first reported EPN in 1898, and Schultz and Klorfein [3] coined the term emphysematous pyelonephritis in 1962 to describe this severe infectious disease. EPN is predominant in female and patients with diabetes mellitus (DM), obstructive uropathy, and immunocompromised conditions, such as previous kidney transplants $[1,4,5]$. In most cases, EPN is caused by gramnegative organisms, such as Escherichia coli, Enterobacter, Klebsiella, and Proteus species [1,4]. The Streptococcus, Candida, Aspergillus, and Cryptococcus species have also been implicated [6-8].

The pathogenicity of EPN has not yet been elucidated fully. On the other hand, bacteria may use the high glucose level in the renal parenchyma as a substrate for fermentation, producing carbon dioxide and hydrogen as byproducts [4]. The ideal management of EPN is unclear. Previously, the conventional treatment for EPN was early nephrectomy and open surgical drainage with antibiotic therapy [9]. On the other hand, this approach has a relatively high mortality rate of $40-50 \%$ [10]. A recent study suggested that EPN should be treated with aggressive medical treatment and often minimally invasive treatment, including percutaneous catheter drainage (PCD) and ureteral stent placement. Prompt surgical intervention should be considered in deteriorating patients [11-13].

EPN is an uncommon condition that many physicians are unlikely to encounter. There is not enough published literature on EPN because of its rarity. This study examined the characteristics, current treatment trends, and outcomes of patients with EPN over a 10-year period in Korea.

\section{MATERIALS AND METHODS}

This study reviewed patients diagnosed with EPN by an abdominal computed tomography (CT) scan between January 2011 and February 2021 at 15 institutes in Korea. Patients with recent abdominal trauma, a history of a fistula between the digestive and urinary tract, and a history of kidneyrelated surgery or procedures were excluded because of their possible effects on the results.
The patients' characteristics, including age, sex, comorbidities (DM, hypertension, stroke, and chronic kidney disease), acute kidney injury, blood and urine tests, and the Huang and Tseng classification, were analyzed. In addition, the treatment modalities (medical, minimally invasive, and surgical therapy) and treatment outcomes (sepsis, septic shock, or mortality) were assessed. The causative organisms and antibiotic resistance profiles were assessed by urine culture, including extended-spectrum $\beta$-lactamase. The 10 -year study period was divided arbitrarily into the following three groups to analyze the trends in mortality rates and treatment of patients with EPN in this period: 2011-2014 (group A), 2015-2017 (group B), and 2018-2021 (group C).

EPN was defined as gas accumulation in the collecting system, renal parenchyma, or perinephric or pararenal space on a CT scan. The Huang and Tseng classification system was used, which is defined as follows: class 1 - gas confined to the collecting system; class 2 - gas confined to the renal parenchyma alone; class $3 \mathrm{~A}$ - perinephric extension of gas or abscess; class $3 \mathrm{~B}$ - extension of gas beyond the Gerota fascia; and class 4 - bilateral EPN or EPN in a solitary kidney. Medical management involved supportive therapy, such as hemodynamic stabilization, metabolic control, and antibiotic therapy. Minimally invasive management involved PCD or an indwelling ureteral stent. Surgical management involved open drainage or a nephrectomy. Sepsis and septic shock were defined according to the third international consensus definitions for sepsis and septic shock (Sepsis-3) [14].

All the variables, which included the categorical and continuous variables, were used for descriptive analyses. The treatment modalities, outcomes, and patient groups were compared using a Pearson's chi-square test. All the analyses were performed using SPSS version 18.0. (SPSS Inc., Chicago, IL, USA).

\section{RESULTS}

After excluding the patients who met the exclusion criteria, 217 patients were included in the final cohort. The mean age of the patients was 65.2 years. The proportion of female patients (74.2\%) was higher than that of the male patients (25.8\%). DM (82.0\%) was the most common comorbidity in the patients, followed by hypertension (48.4\%), chronic kidney disease (30.4\%), and stroke (12.0\%). 
Sixty-six patients (30.4\%) had obstructive uropathy. The most common Huang and Tseng classification was class II (36.9\%), followed in order by class I (31.3\%), class IIIa (16.1\%), class IIIb (9.2\%), and class IV (6.0\%). In urine culture analyses, 143 patients showed positive growth in their urine cultures. E. coli was the most common pathogen in the urine cultures (113 patients, 79.0\%), followed by Klebsiella pneumoniae (19 patients, 13.3\%). The remaining 11 (7.7\%) patients tested positive for Enterococci, Pseudomonas, Candida, Proteus, Morganella, Staphylococcus, and mixed types of bacteria (Fig. 1).

Sepsis was observed in 116 patients (53.5\%). Septic shock occurred in 57 patients (26.3\%). After treatment, the overall mortality rate was $10.6 \%$. The treatment failed in $18.1 \%$ and $26.3 \%$ of the patients with sepsis and septic shock, respectively. Fig. 2 shows the treatment patterns of EPN among the patients included in the study. Of the patients, 95 (43.8\%) were treated with medical management, and 98 (45.2\%) were treated with minimally invasive management. Twenty-four patients (11.0\%) underwent surgical management. The mortality rates of patients who underwent medical, minimally invasive, and surgical management were $13.7 \%, 6.1 \%$, and $16.7 \%$, respectively.

Seventy-one (32.7\%), 66 (30.4\%), and 80 (36.9\%) patients were diagnosed with EPN in 2011-2014 (group A), 2015-2017 (group B), and 2018-2021 (group C), respectively. The proportion of patients who underwent medical management in groups A, B, and C was 46.5\% ( $=33), 47.0 \%(n=31)$, and $38.8 \%$ ( $n=31$ ), respectively. There were no significant changes in the trends across the period. The proportion of patients who underwent minimally invasive management in groups

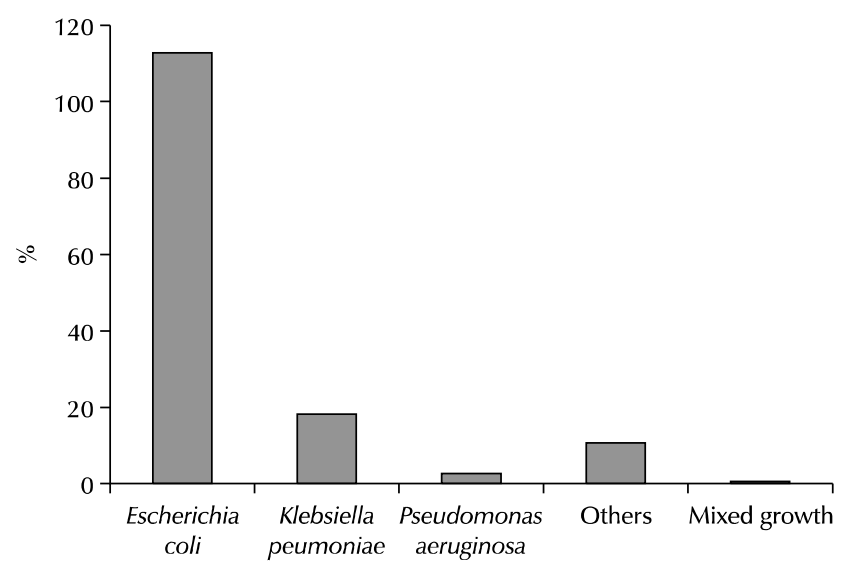

Fig. 1. Causative organisms from urine. Others (Enterococci, Candida, Proteus, Morganella, Staphylococcus species).
A, B, and C was 35.2\% ( $=25), 43.9 \%(n=29)$, and 55.0\% $(n=44)$, respectively. A gradually increasing trend was observed over time. The proportion of patients who underwent surgical management in groups A, B, and C was 18.3\% ( $n=13), 9.1 \%$ $(n=6)$, and $6.3 \%(n=5)$, respectively. A gradually decreasing trend was observed over time. The mortality rates of the patients in groups $\mathrm{A}, \mathrm{B}$, and $\mathrm{C}$ were $7.0 \%, 13.6 \%$, and $11.3 \%$, respectively. No differences in mortality rates were observed between the groups. Table 1 lists the mortality rates of the patients with EPN according to the treatment modalities and time periods. The mortality rate was similar regardless of the treatment modalities and time periods.

\section{DISCUSSION}

EPN is an uncommon disease with a potentially severe and life-threatening clinical course due to septic complications if it is not promptly recognized and treated medically. The reported mortality rate is up to $80 \%$ [1]. In the present results, the demographic characteristics of the patients with EPN were in line with those of previous studies. Similar to other related studies, most patients in the present study were female (74.2\%), and the most common causative pathogen was E. coli, followed by other gram-negative organisms [12,15,16]. Several studies suggested that DM might be the most common risk factor for EPN $[1,4,15,16]$. Similarly, DM (82.0\%) was the most common underlying disease in the present cohort. Obstructive uropathy (30.4\%) was also common among the patients, which was in line with other studies $[1,3,12,16]$.

In the past, the conventional treatment for EPN was early

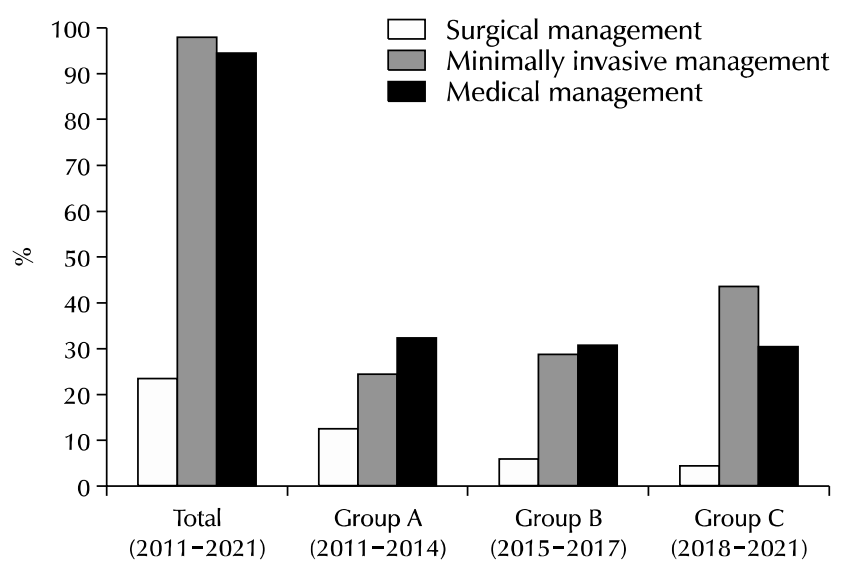

Fig. 2. Analysis of the patterns of emphysematous pyelonephritis treatment among the patients included in the study. 
Table 1. Mortality rate of patients with emphysematous pyelonephritis according to treatment modalities and study period groups

\begin{tabular}{ccccc}
\hline \multirow{2}{*}{ Group } & \multicolumn{3}{c}{ Mortality rate \% (n/total) } \\
\cline { 2 - 5 } & $\begin{array}{c}\text { Overall } \\
(23 / 217)\end{array}$ & $\begin{array}{c}\text { Medical management } \\
(13 / 95)\end{array}$ & $\begin{array}{c}\text { Minimal invasive management } \\
(6 / 98)\end{array}$ & $\begin{array}{c}\text { Surgical management } \\
(4 / 24)\end{array}$ \\
\hline A $(2011-2014)$ & $7.0(5 / 71)$ & $9.1(3 / 33)$ & $0(0 / 25)$ & $15.4(2 / 13)$ \\
B (2015-2017) & $13.6(9 / 66)$ & $19.4(6 / 31)$ & $6.9(2 / 29)$ & $16.7(1 / 6)$ \\
C (2018-2021) & $11.3(9 / 80)$ & $12.9(4 / 31)$ & $9.1(4 / 44)$ & $20.0(1 / 5)$ \\
\hline
\end{tabular}

nephrectomy or open surgical drainage with antibiotic therapy [9]. Klein et al. [17] reviewed 66 cases of EPN. They reported an overall mortality rate of $38 \%$, with only a $29 \%$ chance of survival among those treated medically compared to $71 \%$ among those treated with both medical and surgical treatment. The authors emphasized the early diagnosis and aggressive early combined medical-surgical management for patients with EPN [17]. Huang et al. [1] assessed 48 patients with EPN between 1989 and 1997 and reported that the total mortality rate was $18.8 \%$. Eight of the 14 patients in whom the PCD treatment was unsuccessful underwent a subsequent nephrectomy, seven of whom survived. Only eight patients were managed with direct nephrectomy and survived. The overall success rate for a nephrectomy was $90 \%$. The authors concluded that nephrectomy can provide the best treatment outcome and should be attempted promptly for extensive EPN with a fulminant course [1].

On the other hand, with the advances in imaging technologies, such as CT scanning, image-guided interventional procedures, and antibiotics, the mortality of patients with EPN was reduced when they were treated with antibiotics alone and minimally invasive management.

Chen et al. [10] analyzed 25 patients with EPN who were initially treated with PCD during a 10 -year period. In their study, 20 of the 25 patients (80\%) were treated successfully with antibiotic therapy combined with PCD. The authors concluded that for EPN, antibiotic therapy combined with PCD might be an acceptable alternative to antibiotic therapy with surgical intervention [10]. In another study, the ABACUS Research Group reviewed 10 retrospective studies related to EPN. They reported a $50 \%$ mortality rate from medical management alone, 25\% from medical management combined with emergency nephrectomy, and $13.5 \%$ from medical management combined with PCD. The mortality rate was significantly lower in patients who underwent PCD than in those who underwent other treatments [18]. The authors suggested that PCD should be part of the initial management strategy for EPN [18]. Recently, Karthikeyan et al. [19] reviewed patients with EPN from 2007 to 2014. They reported that 32 out of 34 patients with EPN were treated successfully with conservative management alone, demonstrating a survival rate of $94.1 \%$. All 32 patients were treated successfully with minimally invasive management. Nephrectomy was performed in only two patients. The authors also suggested that medical management with timely intervention with urinary drainage might be effective and curative in most patients [19].

In the current study, 95 (43.8\%), 98 (45.2\%), and 24 (11.0\%) patients underwent medical, minimally invasive, and surgical management, respectively; the corresponding mortality rates were $13.7 \%, 6.1 \%$, and $16.7 \%$. According to these results, the mortality rate of patients treated with medical management combined with PCD was lower than that of patients treated with surgical management (6.1\% vs. $16.7 \%)$. Reflecting these results, the proportion of patients treated with minimally invasive management increased gradually with time (group $\mathrm{A}=35.2 \%$; group $\mathrm{B}=43.9 \%$; group $\mathrm{C}=55.0 \%$ ). On the other hand, the proportion of patients who underwent surgical management tended to decrease gradually with time in the present cohort (group $\mathrm{A}=18.3 \%$; group $\mathrm{B}=9.1 \%$; group $\mathrm{C}=6.3 \%$ ). When the mortality rates were analyzed according to the time period, patients treated with medical management combined with PCD had a lower mortality rate than those treated with surgical management in all time periods (group A: 0\% vs. $15.4 \%$; group B: $6.9 \%$ vs. $16.7 \%$; group C: $9.1 \%$ vs. $20.0 \%)$.

These results suggest that the mortality rate of patients with EPN might be affected by other risk factors, such as the Huang and Tseng classification of EPN, rather than the treatment modality. The mortality rates of class IIIb (25.0\%) and class IV (23.1\%) patients were higher than those of class I-IIIa (5.3-5.3\%) patients.

In the present cohort, the mortality rate of patients with EPN (10.6\%) was relatively lower than that reported in a 
meta-analysis of studies published before the mid-2000s (25\%) [15]. On the other hand, it was similar to that reported in recent studies, reporting mortality rates from $6 \%$ to $12.5 \%$ $[12,16,19]$. These differences might be attributable to advances in imaging technologies, interventional procedures, and antibiotics. In addition, other factors, including ethnicity, regional characteristics, and the medical care system, might have had impacts.

Several studies have investigated patients with EPN, but the number of patients in these studies was relatively small due to the rarity of the disease. To the best of the authors' knowledge, this cohort was the largest for assessing EPN compared to those included in previous studies. A limitation of this study was the risk of potential bias inherent to retrospective studies. On the other hand, the present study consisted of a large, homogeneous, recent group of patients who were evaluated and treated at multiple high-volume centers in Korea. Therefore, these results might better reflect the current EPN treatment trends and outcomes and might also be useful for patient counseling and treatment decision-making for patients with EPN.

\section{CONCLUSIONS}

This study found that EPN had a relatively high treatment success rate with medical management combined with minimally invasive management. The proportion of patients who underwent minimally invasive management tended to increase gradually with time. In contrast, the proportion of those who underwent surgical management tended to decrease gradually with time in Korea. The mortality rate of patients with EPN in the present study was relatively lower (10.6\%) than that in studies published before the 2010s, possibly because of the advances in imaging technologies, medical procedures, and antibiotics.

\section{CONFLICT OF INTEREST}

No potential conflict of interest relevant to this article was reported.

\section{AUTHOR CONTRIBUTIONS}

S.K.C. performed the statistical analysis and wrote the manuscript. K.H.Y. and E.C.H. performed the statistical analysis. J.W.L., S.I.J., E.C.H., J.C., W.B.K., J.S.H., and K.H.Y. review the manuscript. All authors contributed to the acquisition of data. S.B., J.B.C., Y.K., J.M.C., J.H.S., J.H.J., H.C., and T.H.K. participated in the study design and coordination and helped to draft the manuscript. All authors read and approved the final manuscript.

\section{ORCID}

Seung-Kwon Choi, https://orcid.org/0000-0001-5854-8675 Koo Han Yoo, https://orcid.org/0000-0001-7952-7902 Jeong Woo Lee, https://orcid.org/0000-0002-4508-8240 Seung Il Jung, https://orcid.org/0000-0003-4864-8175 Eu Chang Hwang, https://orcid.org/0000-0002-2031-124X Joongwon Choi, https://orcid.org/0000-0001-5978-8179 Woong Bin Kim, https://orcid.org/0000-0002-7369-490X Jung Sik Huh, https://orcid.org/0000-0003-2767-4390 Jin Bong Choi, https://orcid.org/0000-0003-2508-6819 Yeonjoo Kim, https://orcid.org/0000-0001-9656-1678 Jae Min Chung, https://orcid.org/0000-0002-7467-5954 Ju-Hyun Shin, https://orcid.org/0000-0001-5996-5876 Jae Hung Jung, https://orcid.org/0000-0002-4990-7098 Hong Chung, https://orcid.org/0000-0002-0151-4965 Sangrak Bae, https://orcid.org/0000-0002-8364-704X Tae-Hyoung Kim, https://orcid.org/0000-0002-0257-3449

\section{REFERENCES}

1. Huang JJ, Tseng CC. Emphysematous pyelonephritis: clinicoradiological classification, management, prognosis, and pathogenesis. Arch Intern Med 2000;160:797-805.

2. Kelly HA, MacCallum WG. Pneumaturia. JAMA 1898;31:375-81.

3. Schultz EH Jr, Klorfein EH. Emphysematous pyelonephritis. J Urol 1962;87:762-6.

4. Tang HJ, Li CM, Yen MY, Chen YS, Wann SR, Lin HH, et al. Clinical characteristics of emphysematous pyelonephritis. J Microbiol Immunol Infect 2001;34:125-30.

5. Chuang YW, Chen $\mathrm{CH}$, Cheng $\mathrm{CH}$, Hung SW, Yu TM, Wu MJ, et al. Severe emphysematous pyelonephritis in a renal allograft: successful treatment with percutaneous drainage and antibiotics. Clin Nephrol 2007;68:42-6.

6. Pontin AR, Barnes RD, Joffe J, Kahn D. Emphysematous pyelonephritis in diabetic patients. Br J Urol 1995;75:71-4.

7. Turney JH. Renal conservation for gas-forming infections. Lancet 2000;355:770-1.

8. Kamaliah MD, Bhajan MA, Dzarr GA. Emphysematous pyelonephritis caused by Candida infection. Southeast Asian J 
Trop Med Public Health 2005;36:725-7.

9. Michaeli J, Mogle P, Perlberg S, Heiman S, Caine M. Emphysematous pyelonephritis. J Urol 1984;131:203-8.

10. Chen MT, Huang $\mathrm{CN}$, Chou $\mathrm{YH}$, Huang $\mathrm{CH}$, Chiang CP, Liu GC. Percutaneous drainage in the treatment of emphysematous pyelonephritis: 10-year experience. J Urol 1997;157:1569-73.

11. Uruc F, Yuksel OH, Sahin A, Urkmez A, Yildirim C, Verit A. Emphysematous pyelonephritis: our experience in managing these cases. Can Urol Assoc J 2015;9:E480-3.

12. Aboumarzouk OM, Hughes O, Narahari K, Coulthard R, Kynaston $\mathrm{H}$, Chlosta $\mathrm{P}$, et al. Emphysematous pyelonephritis: time for a management plan with an evidence-based approach. Arab J Urol 2014;12:106-15.

13. NanaGR, Brodie A, Akhter W, Karim O, Motiwala $H$. Nephroureterectomy for emphysematous pyelonephritis: an aggressive approach is sometimes necessary. A case report and literature review. Int J Surg Case Rep 2015;10:179-82.

14. Singer $M$, Deutschman CS, Seymour CW, Shankar-Hari $M$, Annane D, Bauer $M$, et al. The third international consensus definitions for sepsis and septic shock (sepsis-3). JAMA 2016;315:801-10.
15. Falagas ME, Alexiou VG, Giannopoulou KP, Siempos II. Risk factors for mortality in patients with emphysematous pyelonephritis: a meta-analysis. J Urol 2007;178(3 Pt 1):880-5 quiz 1129.

16. Lu YC, Chiang BJ, Pong YH, Chen CH, Pu YS, Hsueh PR, et al. Emphysematous pyelonephritis: clinical characteristics and prognostic factors. Int J Urol 201421:277-82.

17. Klein FA, Smith MJ, Vick CW 3rd, Schneider V. Emphysematous pyelonephritis: diagnosis and treatment. South Med J 1986;79:41-6.

18. Somani BK, Nabi G, Thorpe P, Hussey J, Cook J, N'Dow J; ABACUS Research Group. Is percutaneous drainage the new gold standard in the management of emphysematous pyelonephritis? Evidence from a systematic review. J Urol 2008;179:1844-9.

19. Karthikeyan VS, Manohar CMS, Mallya A, Keshavamurthy R, Kamath AJ. Clinical profile and successful outcomes of conservative and minimally invasive treatment of emphysematous pyelonephritis. Cent European J Urol 2018;71:228-33. 